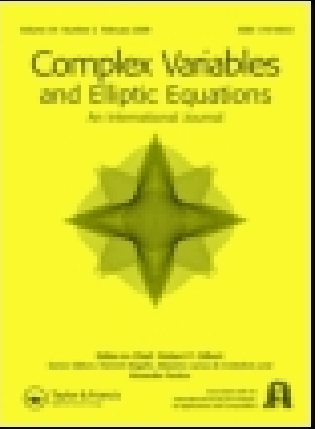

Complex Variables and Elliptic Equations

ISSN: 0278-1077 (Print) 1563-5066 (Online) Journal homepage: https://www.tandfonline.com/loi/gcov19

\title{
On the zero distributionof remainders of entire power series
}

\section{I.V. Ostrovskll}

To cite this article: I.V. Ostrovskll (2001) On the zero distributionof remainders of entire power series, Complex Variables and Elliptic Equations, 43:3-4, 391-397, DOI: $10.1080 / 17476930108815328$

To link to this article: https://doi.org/10.1080/17476930108815328

册 Published online: 26 Jun 2007.

Submit your article to this journal $\widetilde{T}$

Џ Article views: 26 


\title{
On the Zero Distribution of Remainders of Entire Power Series
}

\author{
I.V. OSTROVSKII* \\ Department of Mathematics, Bilkent University, 06533 Bilkent, \\ Ankara, Turkey and B. Verkin Institute for Low Temperature \\ Physics and Engineering, 47 Lenin ave, 61164 Kharkov, Ukraine
}

Communicated by G. C.Wen

Dedicated to the memory of Chi-tai Chuang

(Received 4 January 2000)

It has been shown by the author that, if all remainders of the power series of an entire function $f$ have only real positive zeros, then $\log M(r, f)=O\left((\log r)^{2}\right), r \rightarrow \infty$. The main results of the paper are the following: (i) if at least two different remainders have only real positive zeros, then $\log M(r, f)=O(\sqrt{r}), r \rightarrow \infty$; (ii) this estimate cannot be improved even in the case if one replaces two by any given finite number of remainders.

Keywords: Angular zero distribution; Entire function; Power series; Remainder

AMS Classification Categories: 30D20, 30D10

\section{INTRODUCTION}

Let

$$
f(z)=\sum_{k=0}^{\infty} a_{k} z^{k}
$$

\footnotetext{
* Supported in part by INTAS Grant No. 96-0858.
} 
be a power series with infinite radius of convergence. Let

$$
r_{n}(z)=\sum_{k=n}^{\infty} a_{k} z^{k}
$$

be its $n$th remainder.

In [3] the following theorem has been proved.

THEOREM A If, for all sufficiently large $n$, the remainders $r_{n}$ have only real nonnegative zeros, then

$$
\log M(r, f)=O\left((\log r)^{2}\right), \quad r \rightarrow \infty .
$$

This bound is the best possible in the sense of order.

On the other hand, the following theorem holds.

THEOREM B If there exist two values, $n_{1}$ and $n_{2}$, such that the remainders $r_{n_{1}}$ and $r_{n_{2}}$ are different and have only real nonnegative zeros, then

$$
\log M(r, f)=O(\sqrt{r}), \quad r \rightarrow \infty .
$$

This bound is the best possible in the sense of order.

In a weaker form, this theorem has been proved in [3]; in the above formulation, we will prove it here.

The question arises what will happen when one considering other sets of values of $n$ (than those mentioned in Theorems A and B), for which remainders $r_{n}$ have only real nonnegative zeros. We are going to prove that any finite set of values of $n$ does not imply any better bound, than (3). More precisely, we will prove the following theorem.

THEOREM C Let $N \geq 2$ be an arbitrary integer. There exists an entire function $f$ satisfying

$$
\liminf _{r \rightarrow \infty} \frac{\log M(r, f)}{\sqrt{r}}>0
$$

and such that the remainders $r_{0}, r_{1}, r_{2}, \ldots, r_{N}$ are different and have only real nonnegative zeros. 


\section{MAIN LEMMA}

Denote by $\mathbf{T}$ the class of all real entire transcendental functions $f$ satisfying the condition: there exists a constant $\alpha_{f}>0$ such that all roots of the two equations:

$$
f(z)-\alpha_{f}=0, \quad f(z)+\alpha_{f}=0
$$

are positive.

Lemma If $f \in T$, then there exists a real constant $\beta \neq 0$ such that $z f(z)+\beta \in T$.

Proof We will use the following properties of a function $f \in \mathbf{T}$ :

(i) $f$ is of order not greater than $1 / 2$.

(ii) Zeros $\left\{a_{k}\right\}_{k=1}^{\infty}$ of $f$ and zeros $\left\{b_{k}\right\}_{k=1}^{\infty}$ of $f^{\prime}$ are positive simple and interlace, i.e.,

$$
0<a_{1}<b_{1}<a_{2}<b_{2}<a_{3}<\cdots
$$

(iii) $\left|f\left(b_{k}\right)\right| \geq \alpha_{f}$ for $k=1,2, \ldots$

These properties can be found in $[2, \S 1]$ where reality instead of positivity of roots of (5) is considered.

If $f \in \mathbf{T}$, then $f\left(z^{2}\right)$ belongs to the class considered in [2] therefore (i)-(iii) follow easily.

Without loss of generality we can assume that $f(0)>0$. Then $f$ is decreasing on

$$
\left(-\infty, b_{1}\right] \bigcup\left[b_{2}, b_{3}\right] \bigcup\left[b_{4}, b_{5}\right] \bigcup \cdots
$$

and is increasing on

$$
\left[b_{1}, b_{2}\right] \bigcup\left[b_{3}, b_{4}\right] \bigcup\left[b_{5}, b_{6}\right] \bigcup \cdots
$$

Evidently (see Fig. 1), for sufficiently small $\varepsilon>0$, the number of roots of the equation

$$
f(x)=\frac{\varepsilon}{x}
$$




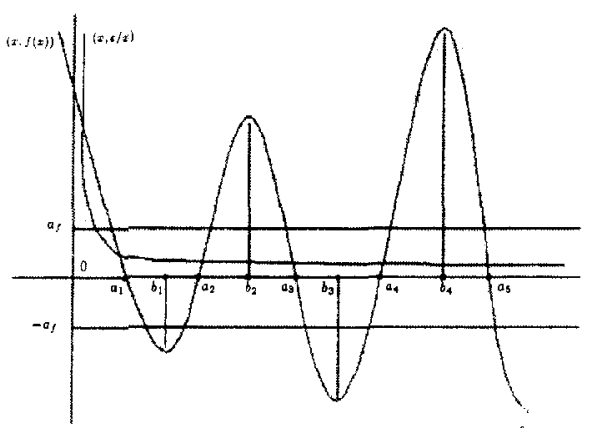

FIGURE 1

lying on the interval $\left[0, b_{k}\right], k=1,2, \ldots$, is not less than $k+1$. Hence the function $z f(z)-\varepsilon$ has at least $k+1$ zeros on $\left[0, b_{k}\right], k=1,2, \ldots$.

Let us consider the rectangle

$$
Q_{k, R}=\left\{z:|\operatorname{Re} z|<b_{k},|\operatorname{Im} z|<R\right\}, \quad k=1,2, \ldots
$$

and show that, for $k$ large enough, we can choose $R$ so large that the following inequality holds

$$
|z f(z)|>\varepsilon, \quad \text { for } z \in \partial Q_{k, R} .
$$

The Hadamard product representation

$$
f(z)=f(0) \prod_{k=1}^{\infty}\left(1-\frac{z}{a_{k}}\right)
$$

implies that $|f(x+i y)|$ is an increasing function of $|y|$ for any fixed $x$. Since $\left|f\left(b_{k}\right)\right| \geq \alpha_{f},\left|f\left(-b_{k}\right)\right|>f(0)$, this implies that $\left|f\left( \pm b_{k}+i y\right)\right| \geq$ $\min \left(\alpha_{f}, f(0)\right)$ for all real $y$ and hence $|z f(z)|>\varepsilon$ on the lines $\{z: \operatorname{Re} z=$ $\left.\pm b_{k}\right\}$ for large enough $k$. Since (7) implies also that $|f(x+i y)| \rightarrow \infty$ as $|y| \rightarrow \infty$ uniformly on any compact set of values of $x$, we can choose $R$ so large that $|z f(z)|>\varepsilon$ on the horizontal sides of $Q_{k, R}$. Thus, (6) holds.

Since the number of zeros of $z f(z)$ in $Q_{k, R}$ is equal to $k+1$, Rouché's theorem implies that all zeros of $z f(z)-\varepsilon$ in $Q_{k, R}$ are positive. We conclude that all zeros of $z f(z)-\varepsilon$ are positive for all sufficiently small $\varepsilon$. 
Let us fix such an $\varepsilon$ and consider $F(z)=z f(z)+\beta$ with $\beta=-\varepsilon / 2$. Then all zeros of both functions $F(z)-|\beta| / 2=z f(z)-3 \varepsilon / 4$ and $F(z)+|\beta| / 2=z f(z)-\varepsilon / 4$ are positive. Thus, $F \in \mathbf{T}$.

\section{PROOF OF THEOREM C}

Firstly, let us do two following remarks. (i) If $f(z)$ and $r_{n}(z)$ are defined by (1) and (2) respectively, then the 'normalized' remainders

$$
t_{n}(z):=z^{-n} r_{n}(z), \quad n=0,1,2, \ldots
$$

satisfy the equation

$$
t_{n-1}(z)=z t_{n}(z)+a_{n-1}, \quad n=1,2, \ldots
$$

(ii) Theorem $\mathrm{C}$ will be proved if we construct an entire function $f$ satisfying (4) and such that $t_{0}, t_{1}, t_{2}, \ldots, t_{N}$ have only nonnegative zeros.

Let us choose as $t_{N}$ any entire function belonging to $\mathbf{T}$ and satisfying (4). For instance, we can choose $t_{N}(z)=\cos \sqrt{z}$. Then, by the main lemma, we choose a real constant $a_{N-1} \neq 0$ such that $t_{N-1}(z)=z t_{N}(z)+a_{N-1}$ belongs to $\mathbf{T}$. Using the lemma once again, we choose $a_{N-2} \neq 0$ such that $t_{N-2}(z)=z t_{N-1}(z)+a_{N-2}$ belongs to $\mathrm{T}$ and so on. Repeating these reasonings, we conclude that the function

$$
f(z)=a_{0}+a_{1} z+a_{2} z^{2}+\cdots+a_{N-1} z^{N-1}+z^{N} t_{N}(z)
$$

has the desired properties.

\section{PROOF OF THEOREM B}

We will prove a more general result. To formulate it, let us consider a finite system of rays

$$
\begin{gathered}
D=\bigcup_{j=1}^{p}\left\{z: \arg z=\alpha_{j}, 0 \leq|z|<\infty\right\}, \\
0 \leq \alpha_{1}<\alpha_{2}<\cdots<\alpha_{p}<2 \pi,
\end{gathered}
$$


and set

$$
\gamma=\min \left\{\alpha_{j+1}-\alpha_{j}: j=1,2, \ldots, p\right\}, \quad \alpha_{p+1}=\alpha_{1}+2 \pi .
$$

THEOREM D Let $f(z)$ be an entire function. Assume that there exist two different polynomials, $P_{1}$ and $P_{2}$, such that all but a finite number of zeros of functions

$$
f-P_{m}, \quad m=1,2,
$$

are located on the system $D$. Then

$$
\log M(r, f)=O\left(r^{\pi / \gamma}\right), \quad r \rightarrow \infty .
$$

We obtain Theorem B by applying Theorem D to the system $D$ consisting of one positive ray $(\gamma=2 \pi)$ and to

$$
P_{m}(z)=\sum_{k=0}^{n_{m}-1} a_{k} z^{k}, \quad m=1,2 .
$$

To prove Theorem $\mathrm{D}$, we apply the following result (see $[1, \mathrm{Ch}$. VI, §2, Theorem 2.4]):

THEOREM Let $F$ be a meromorphic function such that all but a finite number of its zeros and poles are located on a system $D$. If $F$ has a finite nonzero Borel exceptional value, then

$$
T(r, F)=O\left(r^{\pi / \gamma}\right), \quad r \rightarrow \infty .
$$

If $f$ is a transcendental entire function satisfying conditions of Theorem $\mathrm{D}$, then all but a finite number of zeros and poles of the meromorphic function

$$
F(z)=\frac{f(z)-P_{1}(z)}{f(z)-P_{2}(z)}
$$

are located on the system $D$. Moreover, since

$$
F(z)-1=\frac{P_{2}(z)-P_{1}(z)}{f(z)-P_{2}(z)}
$$


the value 1 is Borel exceptional for $F$. Hence, by the theorem quoted above, we have

$$
T(r, F)=O\left(r^{\pi / \gamma}\right), \quad r \rightarrow \infty .
$$

Since $T(r, f)=T(r, F)+O(\log r), r \rightarrow \infty$, we get

$$
\log M(r, f) \leq 3 T(2 r, f)=O\left(r^{\pi / \gamma}\right), \quad r \rightarrow \infty .
$$

\section{References}

[1] Goldberg, A. A. and Ostrovskii, I. V., Distribution of values of meromorphic functions, Moscow, Nauka, 1970 (Russian).

[2] Marchenko, V. A. and Ostrovskii, I. V. (1975). A characterization of the spectrum of Hill's operator. Math. USSR Shornik, 26, 493-554.

[3] Ostrovskii, I. V. (1997). Les séries de puissances dont les restes ont seulement des zéros non-positifs. Comptes Rendus Acad. Sci. Paris, 325, 1257-1262. 